\title{
Self-Adjustment Channel Rank Based on Reordered Cholesky Factorization
}

\author{
Christophe ALEXANDRE, Rabah MAOUDJ, Michel TERRE \\ Lab. CEDRIC / LAETITIA \\ CNAM \\ Paris, France
}

\begin{abstract}
-
This paper presents a self-adjustment of the length of the channel impulse response using a classical Cholesky Factorization. This is of special importance in the context of algorithms containing a matrix inversion and the rank of this matrix is strongly related to the length of the channel impulse, i.e. interferer cancellation algorithm [1].
\end{abstract}

When the length of the channel impulse response is overestimated, the matrix inversion is carried out with a singular matrix. A self-adjustment is then required to avoid an increase in the noise level.

\section{INTRODUCTION}

In most of the low rank channel (number of channel paths is too less than number of pilots) algorithms, the estimation of the true length of the channel impulse response, (the channel impulse response is token at the sampling instants) is important in order to guarantee immunity against the noise.

This case of channel may occur when the sampling time is relatively long, such as in TEDS [2].

Performing interference cancellation algorithm [1], while neglecting the estimation of the length of the channel impulse response means that the performance of the algorithm becomes sub-optimal even when this length of the channel impulse response is assumed much larger than the true one. This problem appears essentially in the matrix inversion included in the algorithm. This matrix becomes obviously singular.

A self-adjustment of the length of the channel impulse response can be achieved using Singular Value decomposition $S V D$ by taking in account only the eigenvectors corresponding to the dominant eigenvalues. The subspace resulting is called commonly the signal-subspace. In [3], several methods to compute eigenvalues/eigenvectors of a positive hermitian matrix are proposed. They mostly use an iterative process exploiting Cholesky decomposition. Pivoting diagonal method $[4,5]$ allows a quick convergence of the process. However for some special cases of matrix, we can make an approximation of these eigenvalues only with the first iteration.

In the present paper, we aim to achieve this self-adjustment with a classical Cholesky after some reordering in the rows and the columns of the matrix to inverse, based on certain assumptions on the channel impulse response.
The rest of the paper is organized as follow. The signal model and interference cancellation technique is described in section II. Section III presents the effect of an over-dimensioned channel response impulse. In section III, the reordered Cholesky technique for self-adjustment of the channel impulse response is detailed. Numerical results of the Cholesky compared to TSVD (Truncated SVD) techniques follow in the next section. Conclusion summarizes the present work in section $\mathrm{V}$.

\section{SIGNAL MODEL AND INTERFERENCE CANCELLATION TECHNIQUE}

In this paper, we consider a two receive antennas SIMO (Single input multiple output) structure with co-channel interferer. The originality of the presented algorithm for cochannel interference cancellation is based on the exploitation of the length of the channel impulse response [1]. The assumption of low rank channel allows the introduction of an extra third weight applied to the reference signal at the receive antennas, see figure 1 .

This algorithm is designed for an OFDM structure with an equidistant comb pilots to achieve the pilot-aided co-channel cancellation and channel equalization.



Signal model received by each antenna is formulated by:

With:

$$
\mathrm{y}_{\mathrm{k}}=\mathrm{h}_{\mathrm{dk}} \mathrm{d}+\mathrm{h}_{\mathrm{zk}} \mathrm{z}+\mathrm{n}_{\mathrm{sk}}, \mathrm{k}=1,2
$$

$$
\begin{array}{ll}
y_{k} & : \text { received signal } \\
d & : \text { useful signal } \\
z & : \text { interfering signal } \\
h_{d k} & : \text { useful channel } \\
h_{z k} & : \text { interfering channel } \\
n_{s k} & : \text { Gaussian noise } \\
k & : \text { index of antenna }
\end{array}
$$

As given in [3], the weights $w_{1}, w_{2}$ are equivalent to a propagation channel and $w_{d}$ to a double of propagation channel. Their impulse responses are $a_{1}, a_{2}$ and $a_{d}$ as given by 
(3). Their estimation is performed by minimizing the mean square error only on the OFDM symbols where the pilots are located (frequency dimension estimation):

$$
\min _{w_{1}, w_{2}, w_{d}}\|e\|^{2}
$$

The solution is given as follow:

With:

$$
\mathrm{a}=\frac{\left(\mathrm{F}_{\mathrm{yd}}^{\mathrm{H}} \mathrm{R}^{\mathrm{H}} \mathrm{RF}_{\mathrm{yd}}\right)^{-1} \mathrm{v}}{\mathrm{v}^{\mathrm{H}}\left(\mathrm{F}_{\mathrm{yd}}^{\mathrm{H}} \mathrm{R}^{\mathrm{H}} \mathrm{RF}_{\mathrm{yd}}\right)^{-1} \mathrm{v}}
$$

$$
R=\left[\begin{array}{lll}
y_{1} & y_{2} & y_{d}
\end{array}\right], F_{y d}=\left[\begin{array}{ccc}
F_{y} & 0 & 0 \\
0 & F_{y} & 0 \\
0 & 0 & F_{d}
\end{array}\right], a=\left[\begin{array}{l}
a_{1} \\
a_{2} \\
a_{d}
\end{array}\right] .
$$

$v=\left[\begin{array}{llllll}0 & \ldots & 0 & 1 & 0 & \ldots\end{array}\right]^{T}$ is the chosen constraint to avoid the null solution.

$F_{y}=F_{L}$ and $F_{d}=F_{2 L}$ are the truncated Fourier basis,

Where:

$$
F_{L}=\frac{1}{\sqrt{N}} e^{-j \frac{2 \pi n L}{N}}
$$

$L$ : supposed length of the channel impulse response.

$N$ : number of pilots in an OFDM symbol.

Finally:

$$
\left\{\begin{array}{l}
\mathrm{w}_{1}=\mathrm{F}_{\mathrm{y}} \mathrm{a}_{1} \\
\mathrm{w}_{2}=\mathrm{F}_{\mathrm{y}} \mathrm{a}_{2} \\
\mathrm{w}_{\mathrm{d}}=\mathrm{F}_{\mathrm{d}} \mathrm{a}_{\mathrm{d}}
\end{array}\right.
$$

These weights are then interpolated to all the OFDM symbols of the frame by a polynomial interpolation.

\section{PROBLEM OF AN OVER-DIMENSIONED CHANNEL IMPULSE RESPONSE}

To avoid the drawback of the complexity imposed by estimating the length of the channel impulse response $L$, it is set often equal to the guard interval GI of the OFDM system in use.

But, it is shown in [6], that the over-dimensioning of $L$ causes an ill estimation of the weights $w_{1}, w_{2}$ and $w_{d}$. This problem appears here as an ill-conditioned matrix $\left(F_{y d}^{H} R^{H} R F_{y d}\right)$ being inverted.

The solution, given in $[6,7]$, consists in adding $\sigma I$ matrix to $\left(F_{y d}^{H} R^{H} R F_{y d}\right)$. This operation is called the Tikhonov regularization $[8,9]$.

Another technique commonly used is TSVD [10]. This method consists in truncating the singular values for $\mathcal{L}<4 L . \mathcal{L}$ is the equivalent regularization parameter .

If $P$ and $S$ are respectively the Eigen vectors and the Eigen values matrices of $\left(F_{y d}^{H} R^{H} R F_{y d}\right)$, then the solution without regularization is:

$$
a=\frac{\sum_{i=0}^{4 \mathrm{~L}} \frac{1}{s_{i i}} p_{i}^{H} v p_{i}}{v^{H} \sum_{i=0}^{4 \mathrm{~L}} \frac{1}{s_{i i}} p_{i}^{H} v p_{i} v}
$$

After regularization it becomes:
With:

$$
a=\frac{\sum_{i=0}^{\mathcal{L}} \frac{1}{s_{i i}} p_{i}^{H} v p_{i}}{v^{H} \sum_{i=0}^{\mathcal{L}} \frac{1}{s_{i i}} p_{i}^{H} v p_{i} v}
$$

$$
\begin{aligned}
& p_{i}: i^{\text {th }} \text { column vector of } P \\
& s_{i i}: i^{\text {th }} \text { diagonal component of } S
\end{aligned}
$$

The high complexity of performing a TSVD is the reason why we introduce this following regularization based on the selfadjustment of the length of the channel impulse response performed by a Cholesky decomposition.

\section{SELF-ADJUSTMENT OF THE LENGTH OF THE CHANNEL IMPULSE RESPONSE BASED ON CHOLESKY DECOMPOSITION}

In the last section, we have seen that when $L$ is overdimensioned, the matrix $\left(F_{y d}^{H} R^{H} R F_{y d}\right)$ becomes singular. In the following, we prove easily that the number of null eigenvalues of a given matrix is detected by Cholesky decomposition.

Let's denote $C$, the Cholesky decomposition of $\left(F_{y d}^{H} R^{H} R F_{y d}\right)$. Then the elements $C_{j i}$ are obtained by:

$$
\begin{aligned}
& \mathrm{c}_{\mathrm{ii}}=\left(\mathrm{r}_{\mathrm{ii}}-\sum_{\mathrm{k}=1}^{\mathrm{i}-1} \mathrm{c}_{\mathrm{ik}}^{2}\right) \\
& \mathrm{c}_{\mathrm{ji}}=\frac{1}{\mathrm{c}_{\mathrm{ii}}}\left(\mathrm{r}_{\mathrm{ij}}-\sum_{\mathrm{k}=1}^{\mathrm{i}-1} \mathrm{c}_{\mathrm{ik}} \mathrm{c}_{\mathrm{jk}}\right)
\end{aligned}
$$

with: $\quad i+1 \leq j \leq 4 L$

$$
r_{i j} \text { are the components of }\left(F_{y d}^{H} R^{H} R F_{y d}\right)
$$

$P$ is the set of Eigen vectors corresponding to the decreasing Eigen values $S$ of the matrix $\left(F_{y d}^{H} R^{H} R F_{y d}\right)$ :

Then

$$
\begin{aligned}
& \left(F_{y d}^{H} R^{H} R F_{y d}\right)=P S P^{H} \\
& C C^{H}=\left(F_{y d}^{H} R^{H} R F_{y d}\right)
\end{aligned}
$$

$$
S=P^{H} C C^{H} P
$$

$$
\text { If } \quad s_{4 L}=0 \quad \text { then } \quad p_{4 L}^{H}(4 L) c_{4 L, 4 L}=0
$$$$
\text { However, } p_{4 L}{ }^{H}(4 L) \neq 0 \text { then } \quad c_{4 L, 4 L}=0 \text {. }
$$

Iteratively, according to equations (6) and (7), the following formula is deduced:

For $0<k \leq 4 L$,

$$
\text { If } \mathrm{s}_{\mathrm{ii}}=0 \text { then } \mathrm{c}_{\mathrm{ii}}=0, k \leq i \leq 4 L
$$

To achieve the self-adjustment of the length of the channel impulse response we have to know the power characteristic of propagation channel paths. This characteristic allows us to neglect the paths of near zero power.

The mean power $\Theta_{i}$ of the propagation channel path $i$ at the sampling instant is related to its propagation delay $\tau_{i}$ (when taking in consideration only the path-loss and the multipath in propagation channel) by the formula of the power delay profile. It is approximated by the following formula [11, 12, 13]. 


$$
\mathrm{E}\left[\Theta_{\mathrm{i}}\right] \propto \frac{\Theta_{0}}{\tau_{\mathrm{i}}^{\alpha}}
$$

With: $\quad \alpha \geq 2$ and $0 \leq i<L$.

$\Theta_{0}$ a constant power.

$E\left[\Theta_{i}\right]$ denotes the mathematical expectation of $\Theta_{i}$.

This assumption means that by analogy to the propagation channel paths, the mathematical expectation of the modulus of the components $a_{1 i}, a_{2 i}, a_{d i}$ follows an exponentially decreasing curve.

Exploiting this result, we can reorder the elements of vector $a$ according to the formula (9). Therefore $a$ becomes $a_{o}$ after this operation:

$$
a=\left[\begin{array}{c}
a_{11} \\
a_{12} \\
\vdots \\
a_{1 L} \\
a_{21} \\
\vdots \\
a_{2 L} \\
a_{d 1} \\
\vdots \\
a_{d 2 L}
\end{array}\right] \text { becomes } a_{o}=\left[\begin{array}{c}
a_{11} \\
a_{21} \\
a_{d 1} \\
a_{12} \\
a_{22} \\
a_{d 2} \\
a_{d 3} \\
\vdots \\
a_{d 2 L-1} \\
a_{d 2 L}
\end{array}\right]
$$

Before proceeding to Cholesky factorization, the columns and rows of $\left(\mathrm{F}_{\mathrm{yd}}^{\mathrm{H}} \mathrm{R}^{\mathrm{H}} \mathrm{RF}_{\mathrm{yd}}\right)$ are subjected to the same order as in $\mathrm{a}_{\mathrm{o}}$. The decomposition process is interrupted when the value of $c_{i i}$ is less than a pre-determined threshold (generally the threshold is a bit superior to the noise power). We denote the value of $\mathrm{i}$ by $\mathcal{L}=\mathrm{i}$ when the decomposition process is exited $(\mathcal{L} \leq 4 \mathrm{~L})$.

If $\mathcal{L}<4 \mathrm{~L}$, the lower triangular matrix $\mathrm{C}$ contains only $\mathcal{L}$ rows of the full Cholesky decomposition. This means that some of the latest component of $a_{0}$ are set to zero and the length of the channel impulse response is self-adjusted.

\section{NUMERICAL RESULTS}

The following results depicted by figures 2 to 7 are obtained by simulating, in full-precision format, the interference cancellation algorithm described above.

The propagation channel used for the useful and interferer signals are $T U 50$ Typical Urban with $50 \mathrm{~km} / \mathrm{h}$ velocity (2 paths) and $H T 200$ Hilly Terrain with $200 \mathrm{~km} / \mathrm{h}$ velocity (3 paths) [14].

The reordered Cholesky results are compared to FLIR the fixed length of the impulse response $L=\mathrm{GI}=4$ (guard interval length) and to TSVD at several exit threshold levels.

For all the considered thresholds, the performance of both Cholesky and TSVD are better than the FLIR when $L=\mathrm{GI}$. This result is expected because $L=4$ is over dimensioned for both channel propagation TU5O and HT200 where for an optimum performance $L$ must be equal to 2 and 3 respectively.

When the threshold becomes high, the performance obtained by Cholesky method tends to improve. We can observe that the performance of this method is monotonically improved with an increase of the threshold.

Unlike Cholesky method, the performance of TSVD seems to be insensitive to the threshold changes.

For such cases of propagation environments and interference/noise power, the optimal value of the threshold can be set to $1.5 \sigma_{\mathrm{n}}{ }^{2}$ where $\sigma_{\mathrm{n}}{ }^{2}$ is the noise power, see figure. 6 and 7.

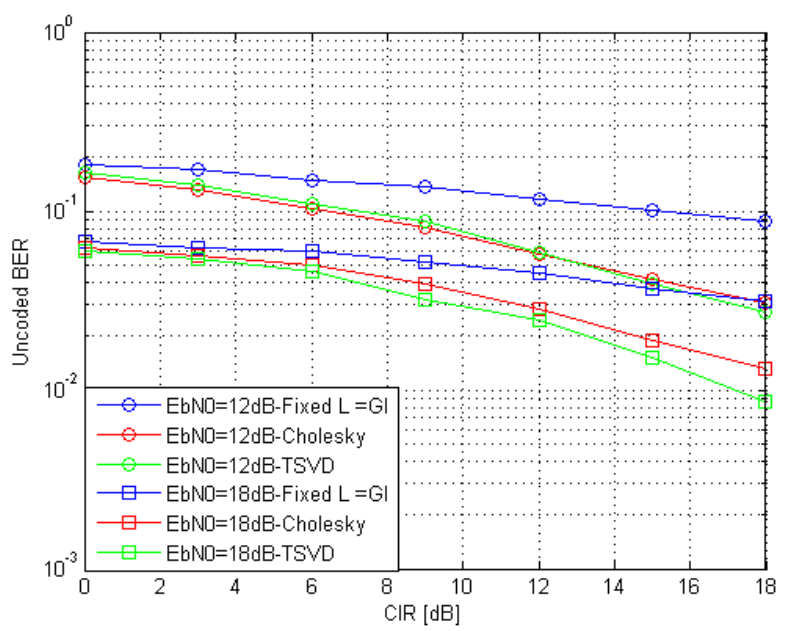

Figure 2. TU50: Uncoded BER vs CIR For treshold $=\sigma_{n}{ }^{2}$



Figure 3. HT200: Uncoded BER vs CIR For treshold $=\sigma_{n}{ }^{2}$



Figure 4. TU50: Uncoded BER vs CIR For treshold $=1.25 \sigma_{n}{ }^{2}$ 


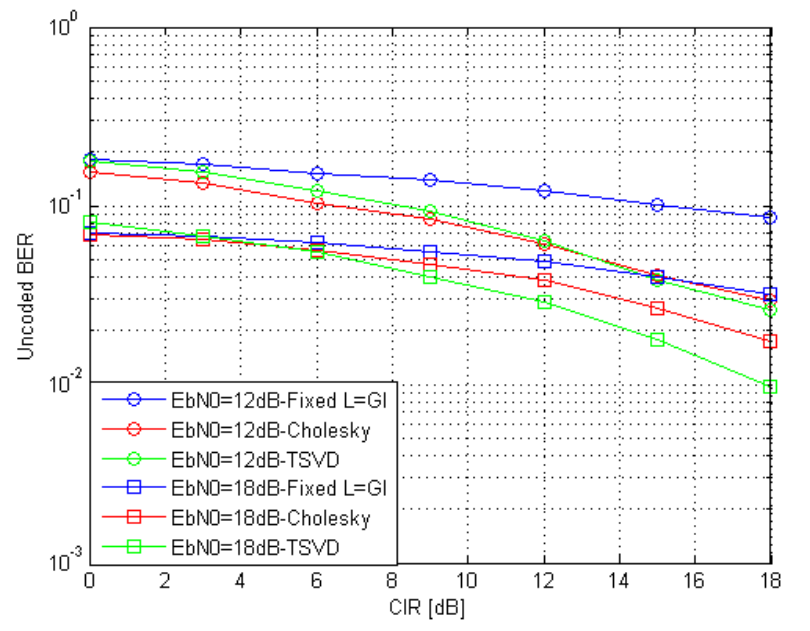

Figure 5. HT200: Uncoded BER vs CIR For treshold $=1.25 \sigma_{n}{ }^{2}$



Figure 6. TU50: Uncoded BER vs CIR For treshold $=1.5 \sigma_{n}{ }^{2}$

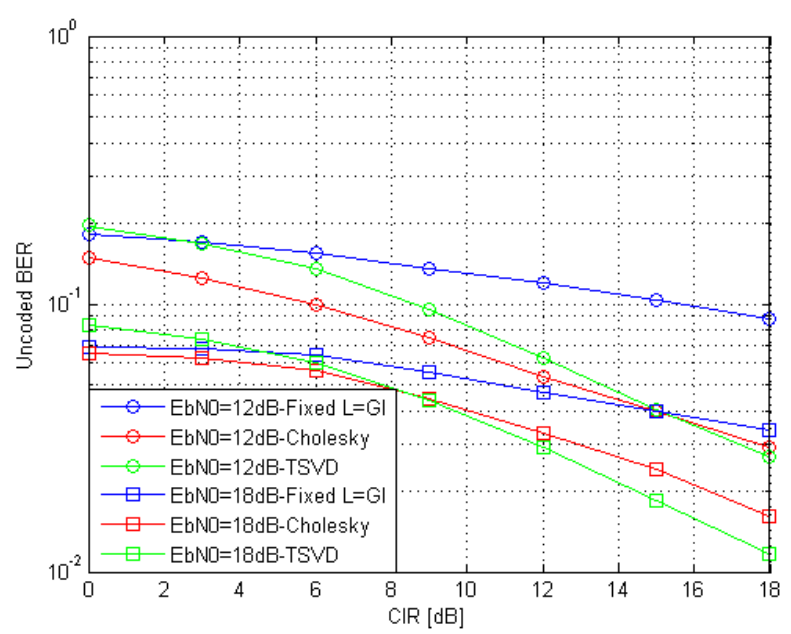

Figure 7. HT200: Uncoded BER vs CIR For treshold $=1.5 \sigma_{n}{ }^{2}$

\section{CONCLUSION}

In this paper, a regularization method for an interference cancellation algorithm based on the reordered Cholesky factorization is studied. According to the obtained results, for low $C I R$, this method seems to be more efficient than the TSVD for an appropriate decomposition process exit threshold level.
In addition, the reordered Cholesky presents low complexity and low execution time, which makes it, more interesting compared to TSVD.

\section{REFERENCES}

[1] L. Fety, R. Maoudj, M. Terre, "Reduced Rank Spatial Filter for Interference Cancellation", submitted to ISWCS 2012.

[2] M. Nouri, V. Lottici, R. Reggiannini, "TEDS : A high speed digital mobile communication air interface for professional users," IEEE Vehicular Technology Magazine, vol 1, issue 4, pp 32-42, December 2006.

[3] R. R. Holmes,H. Huanh, and T. Tam, “Asymptotic Behavior of Iwasawa and Cholesky Iterations", , Linear Algebra and its Applications (May 2011).

[4] R. B. Schnabel and E. Eskow, "A new modified Cholesky factorization", SIAM J. Sci. and Statist. Comput., vol. 11 (1990), pp. 1136-1158.

[5] A. Forsgren, P. E. Gill, and W. Murray, "Computing Modified Newton Directions Using a Partial Cholesky Factorization”, SIAM J. Sci. Comput., Vol. 16, No. 1, pp. 139-150.

[6] Christophe ALEXANDRE, Rabah MAOUDJ, Denis POPIELSKI, Michel TERRE, "DSP Implementation of Interference Cancellation Algorithm for a SIMO System", submitted to ISWCS 2012.

[7] M. A. Lagunas, J. Vidal, A. I. Pérez Neira, "Joint Array Combining and MLSE for single-User Receivers in Multipath Gaussian Multiuser Channels," IEEE Journal on Selected Areas in Communication, vol. 18, no 11 , November 2000.

[8] Tikhonov AN, Arsenin VI. Solutions of ill-posed problems. Washington/ New York; Winston, distributed by Halsted Press. 1977.

[9] L. Hu, X. Zhou, L. Zhang, "Blind Multiuser Detection Based on Tikhonov Regularization”, IEEE Communications Letters, vol. 15, no. 5, May 2011.

[10] G. Shou, L Xia, M. Jiang, Q. Wei, F. Liu, S. Crozier, "Truncated Total Least Squares: A New Regularization Method for the Solution of ECG Inverse problems," IEEE Transactions On Biomedical Engineering, vol. 55, no. 4, April 2008.

[11] Hata M. Et al, "Empirical Formula for Propagation Loss in Land Mobile Radio Services,” IEEE Trans. onVehicular Technology, VT-29, 1980.

[12] Okumura Y. Et al, "Field strength and its variability in VHF and UHF land-mobile radio service", Rev.Elec.Comm. Lab., NTT, Vol.16,9-10, 1968.

[13] J. Mullen, Hong Huang, "Impact of multipath fading in wireless ad hoc networks", Proceedings of the 2nd ACM international workshop on Performance evaluation of wireless ad hoc, sensor, and ubiquitous networks, NY, USA, 2005.

[14] COST-207: "Digital land mobile radio communications," Final report of the COST-Project 207, Commission Of the European Community, Brussels, 1989. 